\title{
Cdc20 inhibitor apcin inhibits the growth and invasion of osteosarcoma cells
}

\author{
YUAN GAO, BENYUAN ZHANG, YUMING WANG and GUANNING SHANG
}

\begin{abstract}
Department of Orthopaedics, Cancer Hospital of China Medical University, Liaoning Cancer Hospital and Institute, Shenyang, Liaoning 110042, P.R. China
\end{abstract}

Received December 6, 2017; Accepted May 22, 2018

DOI: $10.3892 /$ or.2018.6467

\begin{abstract}
Osteosarcoma (OS) is the most common primary malignant bone tumor worldwide, primarily affecting children and adolescents. The anaphase promoting complex/cyclosome (APC/C) is a ubiquitin ligase that is activated by its co-activator Cdc20 during the metaphase-anaphase transition. Apcin is a novel cell-permeable molecule that blocks the interaction between APC/C and Cdc20. Cdc20 overexpression has been reported in various malignancies and plays an oncogenic role in tumorigenesis and tumor progression. In the present study, the antitumor properties of apcin, an inhibitor of Cdc20, was investigated in OS cell lines. In addition, the possible molecular target by which apcin mediates cell death was explored. Apcin was demonstrated to inhibit OS cell growth and induce significant apoptosis. The invasion and mobile abilities of OS cells were also markedly suppressed by apcin treatment. Furthermore, Bim and p21 were upregulated in OS cells following apcin treatment. The results of the present study indicated that apcin may have therapeutic potential as a treatment for OS and that Cdc20 may be a promising molecular target for chemotherapy.
\end{abstract}

\section{Introduction}

Osteosarcoma (OS) is the most common primary malignant bone tumor worldwide, predominantly affecting children and adolescents (1). OS typically presents between the ages of 10 and 20 years or in elderly patients $(2,3)$ and generally develops from primitive mesenchymal bone-forming cells in the long bones (4). At present, the therapeutic options for OS are surgery and intensive multi-agent chemotherapy, including cisplatin, doxorubicin, ifosfamide and methotrexate (5). The 5 -year survival rate of patients with OS without metastases is $60-70 \%$, depending on the use of chemotherapy (6). However,

Correspondence to: Dr Guanning Shang, Department of Orthopaedics, Cancer Hospital of China Medical University, 44 Small River Road, Shenyang, Liaoning 110042, P.R. China

E-mail: shangguanning@hotmail.com

Key words: osteosarcoma, Cdc20, cell growth, invasion, migration the 5-year survival rate for OS patients with metastases, especially pulmonary metastases, is only $20 \%$ (7). The current treatment strategies for metastatic OS typically result in poor prognoses and relapse (8). It would, therefore, be beneficial to develop novel therapeutic agents and innovative treatment approaches to improve survival rates in patients with OS.

The ubiquitin-proteasome system (UPS) is highly regulated and plays major roles in several biological processes. UPS controls different protein functions and important processes associated with the cell cycle, proliferation, metabolism, apoptosis and survival by targeting substrates for ubiquitination and degradation (9). The anaphase promoting complex/cyclosome (APC/C) is an unusually large multimeric cullin-RING ubiquitin ligase that plays a role in the cell cycle. RING finger E3 ubiquitin ligase cell division cycle 20 homolog (Cdc20) serves as an activator of $\mathrm{APC} / \mathrm{C}$ during the metaphase-anaphase transition (10-12). High levels of Cdc20 have been reported in a number of malignancies and are associated with tumorigenesis and tumor progression $(13,14)$. It has previously been reported that $\mathrm{Cdc} 20$ overexpression is associated with the occurrence of glioblastomas, while Cdc20 downregulation occurs in patients with low-grade tumors (15). Recently, Mao et al (16) demonstrated that Cdc20 overexpression may serve as an independent predictor of biochemical recurrence in patients with clinically localized prostate cancer undergoing laparoscopic radical prostatectomy without neoadjuvant therapy. Furthermore, $\mathrm{Cdc} 20$ upregulation was reported to be associated with poor prognosis in urothelial bladder (17), uterine cervix (18), colorectal cancer (19), pancreatic ductal adenocarcinoma (20), oral squamous cell carcinoma (21), gastric (22) and lung cancer (23). These findings revealed that Cdc20 may be a promising novel therapeutic target for cancer treatment (13). One study revealed that small interfering (si)RNA-mediated Cdc20 knockdown suppressed metastatic castration-resistant prostate cancer growth and enhanced chemosensitivity to docetaxel (24). Recently, Zhang et al (25) revealed that polyphenol curcumin inhibited pancreatic cancer cells via downregulation of the expression of $\mathrm{Cdc} 20$.

The APC/C inhibitor apcin is a small novel cell-permeable molecule that blocks the interaction between APC/C and $\mathrm{Cdc} 20$ or Cdh1 (26). It is accepted that apcin prevents substrate recognition by binding to $\mathrm{Cdc} 20$. Furthermore, apcin has been reported to induce metaphase arrest and apoptotic cell death in multiple myeloma (27). de Lange et al (28) demonstrated 
that several cancer cell lines with cohesion fatigues exhibited an increased response to apcin, indicating that APC/C-Cdc20 inhibitors may be effective therapeutic agents targeting cohesion defective cancers. However, the antitumor properties of apcin in OS have not been previously investigated. The aim of the present study was to determine whether apcin exhibited its antitumor properties in a human OS cell line. The possible molecular target that regulated cell death was also investigated.

\section{Materials and methods}

Cell culture. Human osteosarcoma cell lines MG63 and U2OS were purchased from the Chinese Academy of Sciences (Shanghai, China) and maintained in Dulbecco's modified Eagle's medium (DMEM; Gibco; Thermo Fisher Scientific, Inc., Grand Island, NY, USA), supplemented with $10 \%$ fetal bovine serum (FBS; Gibco; Thermo Fisher Scientific, Inc.) and $1 \%$ penicillin/streptomycin (HyClone ${ }^{\mathrm{TM}}$; GE Healthcare Life Sciences, Logan, UT, USA) at $37^{\circ} \mathrm{C}$ with $5 \% \mathrm{CO}_{2}$.

Reagents and antibodies. MTT (3-4,5-dimethyl-2-thiazolyl-2,5-diphenyl-2-H-tetrazolium bromide) and Calcein-AM were purchased from Sigma-Aldrich; Merck KGaA (St. Louis, MO, USA). MTT and Calcein-AM were diluted in dimethyl sulfoxide (DMSO) and stored at $-20^{\circ} \mathrm{C}$, respectively. Primary antibodies for Bim [B-cell lymphoma 2 (Bcl-2) interacting mediator of cell death] (dilution 1:1,000; cat. no. 2933) and p21 (dilution 1:1,500; cat. no. 2947) were purchased from Cell Signaling Technology, Inc. (Danvers, MA, USA). Monoclonal anti-tubulin (dilution 1:3,000; cat. no. T9028) was purchased from Sigma-Aldrich. Secondary antibodies (dilution 1:2,500; cat. no. A-11031; dilution 1:3,000; cat. no. A-11034) were purchased from Thermo Fisher Scientific, Inc. (Waltham, MA, USA).

MTT assay. MG63 and U2OS (2.5×10³ cells/well) were seeded in 96-well plates and cultured overnight. Different concentrations of apcin were added to the medium and cultured for 24, 48 and 72 h, respectively. An MTT assay was then carried out to assess the cell viability according to the manufacturer's protocols. Then, $10 \mu \mathrm{l}$ of MTT solution $(0.5 \mathrm{mg} / \mathrm{ml})$ was added to each well followed by incubation for $4 \mathrm{~h}$. The liquid supernatant was then drained off and $100 \mu \mathrm{l}$ of DMSO was added to each well to dissolve the crystals. The absorbance of each well at $490 \mathrm{~nm}$ was determined using a Multimode Reader of SpectraMax M5 (Molecular Devices, Sunnyvale, CA, USA).

Cell apoptosis assay. MG63 and U2OS cells ( $2 \times 10^{5}$ cells/well) were seeded in 6-well plates and treated with different concentrations of apcin for $48 \mathrm{~h}$. Then, the cells were harvested and washed with phosphate-buffered saline (PBS). Subsequently, the cells were resuspended in $500 \mu \mathrm{l}$ binding buffer with $5 \mu \mathrm{l}$ propidium iodide (PI) and $5 \mu \mathrm{l}$ FITC-conjugated anti-Annexin $\mathrm{V}$ antibody. The apoptosis was analyzed by a flow cytometer (FACScalibur; BD Biosciences, San Jose, CA, USA).

Transwell assays. MG63 and U2OS cells were suspended at a density of $3 \times 10^{4}$ cells/well in $200 \mu \mathrm{l}$ of DMEM. The cell suspension was placed on the upper layer of a cell permeable membrane in Matrigel-precoated Transwell inserts (24-well insert; Corning Inc., Corning, NY, USA) or inserts that were not coated with Matrigel. A total of $500 \mu \mathrm{l}$ of DMEM containing apcin was added in the lower-chamber below the cell permeable membrane. Following incubation for $\sim 16 \mathrm{~h}$, the cells that had migrated and invaded, respectively through the membrane were stained with crystal violet or Calcein-AM, and then images were captured using an inverted microscope (Olympus IX71; Olympus Corp., Tokyo, Japan).

Wound healing assay. MG63 and U2OS cells ( $1 \times 10^{5}$ cells $\left./ \mathrm{ml}\right)$ were seeded on a 6-well plate and incubated till the cell monolayer grew to almost $90 \%$ confluence. Directional cell migration was determined by creating a rectangular wound in a monolayer using a sterile $100-\mu 1$ pipette tip. The open gap was inspected microscopically over a period of time as cells migrated and filled the wound. Photographic images were captured at the beginning and at $20 \mathrm{~h}$ using an inverted microscope (Olympus IX71; Olympus Corp.). The images were compared to quantify the migration rate of the cells.

Western blot analysis. Apcin-treated MG63 and U2OS cell samples were dissociated in cell lysis buffer supplemented with protease inhibitors. A BCA assay (Beijing Solarbio Science and Technology, Co., Ltd., Beijing, China) was performed to quantify the protein concentrations. Equal protein samples were boiled for $5 \mathrm{~min}$ in $1 \mathrm{X}$ loading buffer containing sodium dodecyl sulfate (SDS). The negatively charged protein samples were then separated by $10 \%$ SDS-PAGE gel. Following electrophoretic separation, separated proteins were transferred to a polyvinylidene difluoride membrane (PVDF; EMD Millipore, Billerica, MA, USA). The membrane was blocked with $5 \%$ non-fat milk for $1 \mathrm{~h}$ to prevent non-specific antibody binding and subsequently probed with appropriate primary antibodies specific to the target proteins overnight at $4{ }^{\circ} \mathrm{C}$. The membrane was washed with TBST and incubated with a secondary antibody for $1 \mathrm{~h}$ at room temperature. Finally, an electrochemiluminescence (ECL; Sigma-Aldrich, St. Louis, MO, USA) assay was performed to visualize the protein bands. Quantitative analysis was carried out using QuantiOne imaging software with gel imaging equipment (Bio-Rad Laboratories, Inc., Hercules, CA, USA).

Statistical analysis. Three replications were performed for each experiment. All of the data was analyzed by one-way analysis of variance (ANOVA) and Dunnett's post hoc test using GraphPad Prism 4.0 (Graph Pad Software, Inc., La Jolla, CA, USA). All statistical data were presented as the mean \pm SD of triplicate determinants. $\mathrm{P}<0.05$ was considered to indicate a statistically significant result.

\section{Results}

Apcin suppresses cell proliferation. We first determined whether the Cdc20 inhibitor apcin suppressed human OS cell proliferation. An MTT assay was performed for MG63 and U2OS cells following treatment with the designated concentrations of apcin for 24, 48 and $72 \mathrm{~h}$, respectively. The results revealed that OS cell proliferation was significantly suppressed by apcin in a time- and dose-dependent manner (Fig. 1). 
A

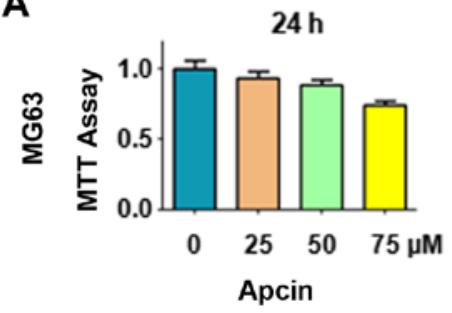

B

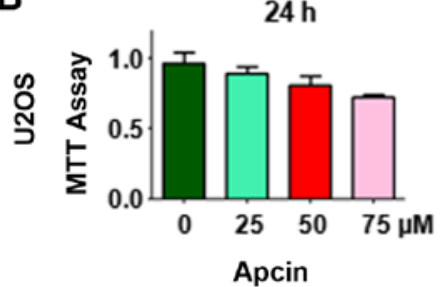

$48 \mathrm{~h}$

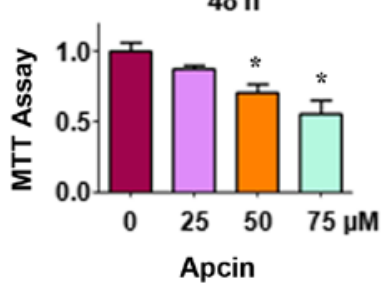

$48 \mathrm{~h}$

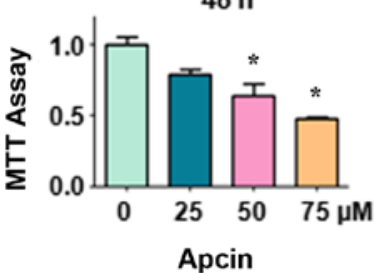

$72 \mathrm{~h}$

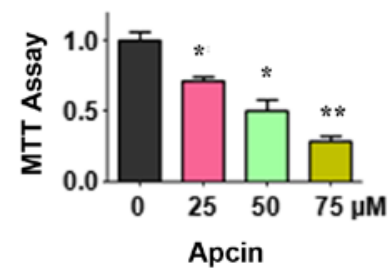

$72 \mathrm{~h}$

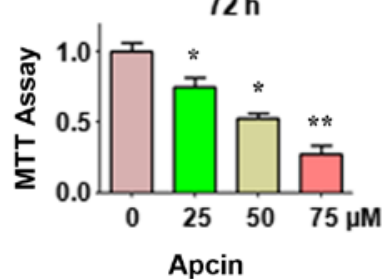

Figure 1. Apcin suppresses human osteosarcoma cancer cell growth. An MTT assay was performed to detect osteosarcoma cancer cell growth following treatment with apcin. Briefly, (A) MG63 cells and (B) U2OS cells were seeded in 96-well plates, cultured overnight and treated with different concentrations of apcin for 24,48 or $72 \mathrm{~h}$, respectively. Then, $10 \mu \mathrm{l}$ MTT solution $(0.5 \mathrm{mg} / \mathrm{ml})$ was added to each well for $4 \mathrm{~h}$. The supernatant was drained off and $100 \mu 1$ dimethyl sulfoxide was added to dissolve the formazan crystals. The absorbance of each well was measured at $490 \mathrm{~nm}$. ${ }^{*} \mathrm{P}<0.05$ and ${ }^{* *} \mathrm{P}<0.001 \mathrm{vs}$. the control groups.
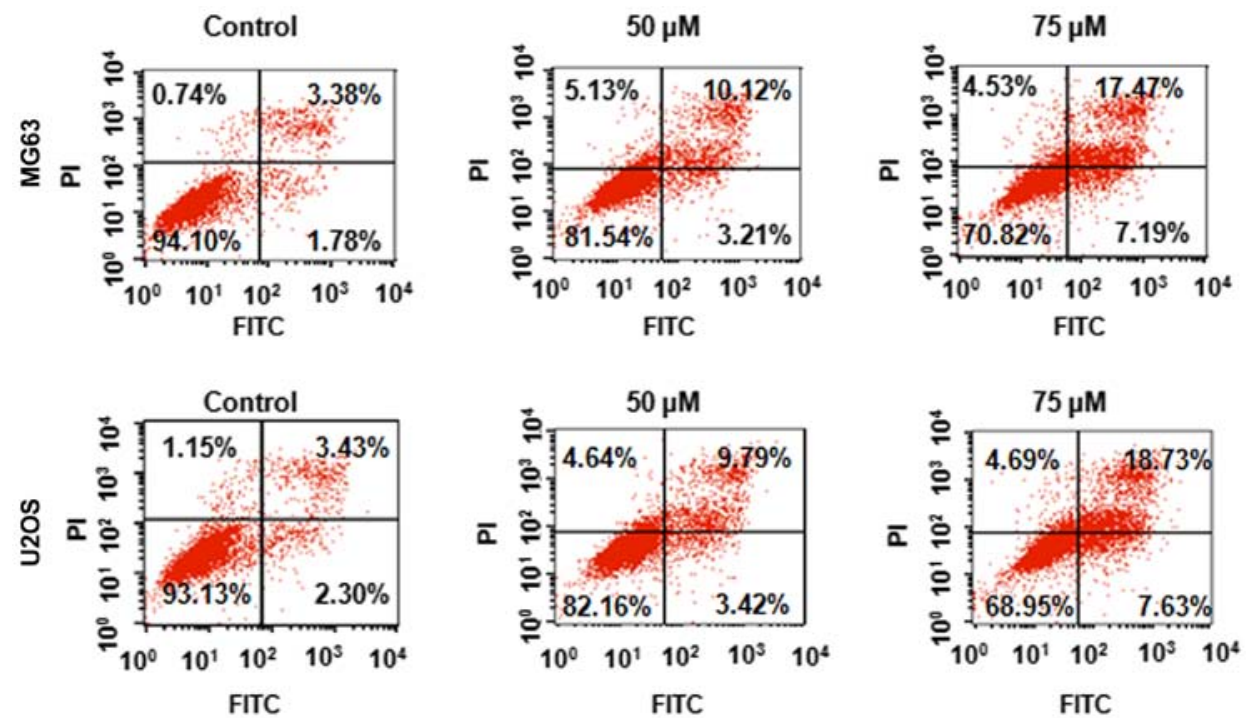

Figure 2. Apcin induces osteosarcoma cell apoptosis. MG63 and U2OS apoptosis was assessed using flow cytometry following treatment with apcin. Briefly, MG63 and U2OS cells were seeded in 6-well plates and treated with different concentrations of apcin for 48 h. Cells were harvested, washed with PBS and resuspended in binding buffer containing PI and FITC-conjugated anti-Annexin V antibody. Apoptosis was analyzed using a flow cytometer.

Treatment with $25 \mu \mathrm{M}$ apcin caused slight OS cell growth inhibition at 24 and $48 \mathrm{~h}$, but $25 \%$ inhibition at $72 \mathrm{~h}$. However, treatment with 50 or $75 \mu \mathrm{M}$ apcin led to $\sim 50$ and $\sim 75 \%$ cell growth inhibition, respectively, at $72 \mathrm{~h}$ in both MG63 and U2OS cells. These results demonstrated that apcin exhibited its antitumor characteristics in human OS cells.

Apcin induces apoptosis. It was further investigated whether apcin affected apoptosis in human OS cells. MG63 and U2OS cells were treated with the designated concentrations of apcin for $48 \mathrm{~h}$ and cell apoptosis was assessed using a PI/FITC-Annexin V assay. We revealed that apcin treatment induced significant cell apoptosis in a dose-dependent manner (Fig. 2). These results indicated that apcin inhibited human OS cell proliferation by inducing apoptosis.
Apcin inhibits Transwell cell migration and invasion. Thereafter, we determined whether apcin reduced OS cell migration and invasion. Cells were stained with crystal violet or Calcein-AM and Transwell migration and invasion assays were performed. The results revealed that apcin treatment suppressed the migration in MG63 cells (Fig. 3). In addition, it was revealed that apcin treatment suppressed the invasion of both MG63 and U2OS cells through the pores of the Matrigel-coated membrane (Fig. 4). Furthermore, the inhibition of migration and invasion was demonstrated to be inhibited in a dose-dependent manner.

Apcin inhibits cell migration in a wound healing assay. We also examined whether apcin suppressed OS cell migration. Both MG63 and U2OS cells were treated with apcin and a 


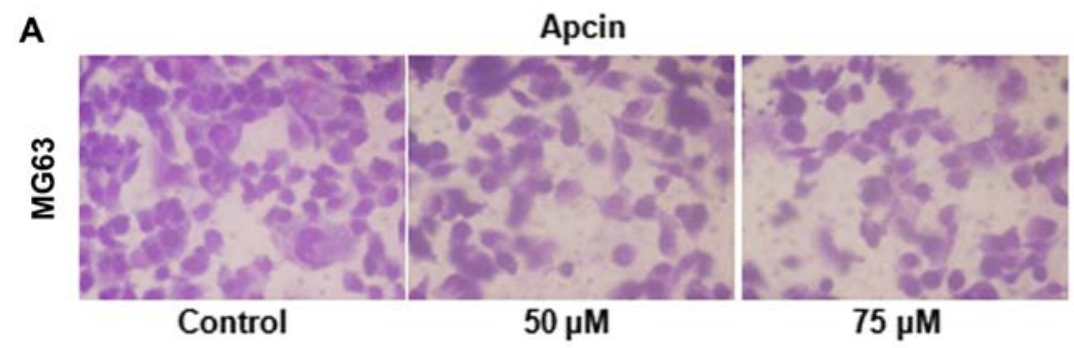

B

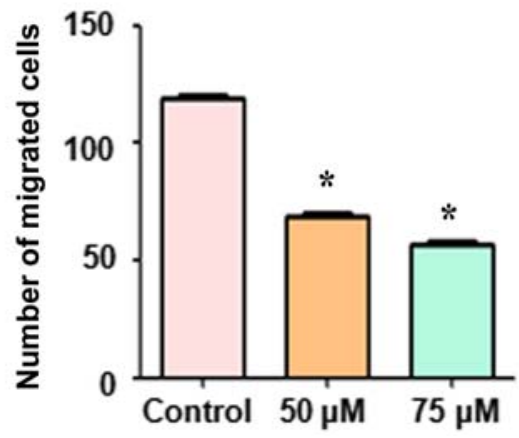

Figure 3. Apcin suppresses the migration of osteosarcoma cancer cells. (A) A Transwell assay was performed to assess the effect of apcin on the migration potential of MG63 cells. Cells that migrated through the membrane were stained with crystal violet and images were captured under a microscope. (B) Quantitative results of A. ${ }^{*}<0.05$ vs. the control group.

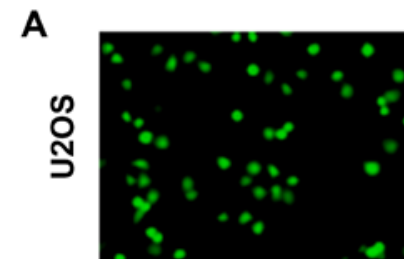

Control

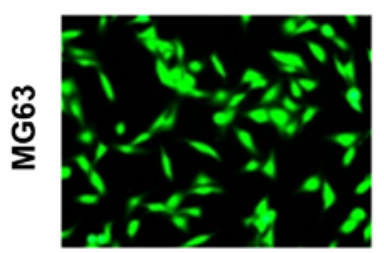

Control

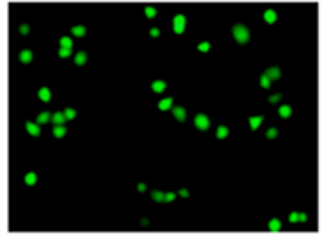

$50 \mu \mathrm{M}$

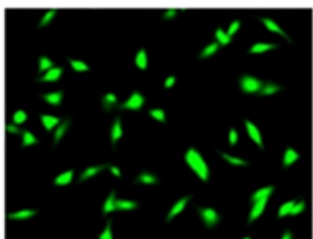

$50 \mu \mathrm{M}$

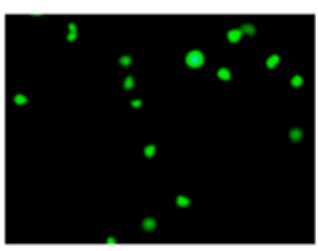

$75 \mu \mathrm{M}$

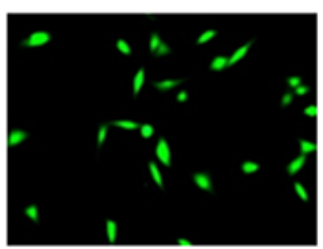

$75 \mu \mathrm{M}$
B

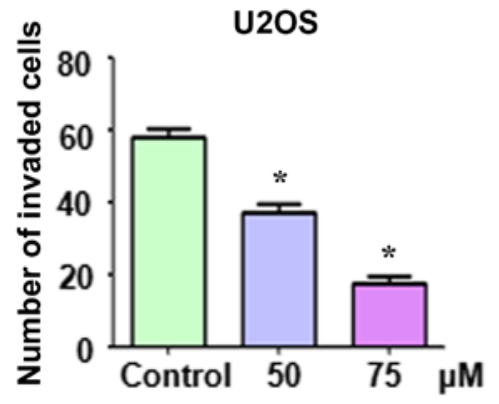

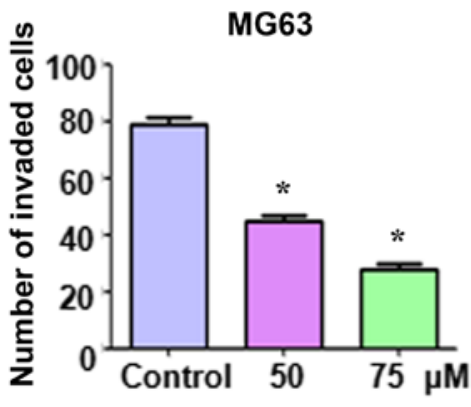

Figure 4. The invasion of MG63 and U2OS cells is inhibited in a dose-dependent manner. (A) Briefly, MG63 and U2OS cells treated with apcin were seeded in the upper chamber of Matrigel-precoated Transwell inserts. A total of $500 \mu 1$ DMEM containing 10\% FBS was added in the lower-chamber. Following incubation for $16 \mathrm{~h}$, invaded cells were stained with Calcein-AM. Images were captured under a microscope. (B) Quantitative results of A. ${ }^{*} \mathrm{P}<0.05$ vs. the control group.

wound healing assay was performed. It was observed that apcin treatment markedly suppressed OS cell migration in a dose-dependent manner (Fig. 5).
Apcin induces Bim expression. The pro-apoptotic molecule Bim has attracted increasing attention as a possible target for tumor therapy. In the present study, we determined whether 


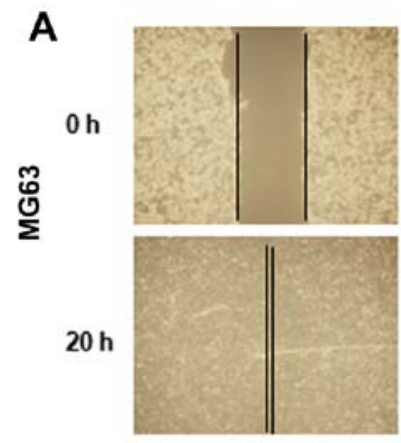

Control

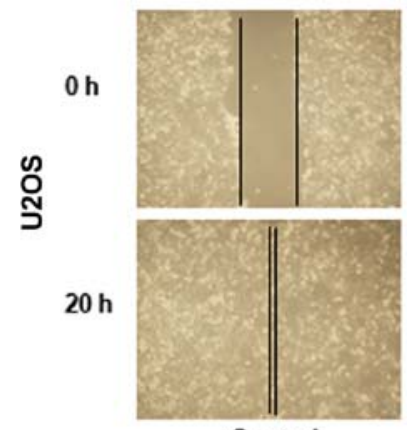

Control

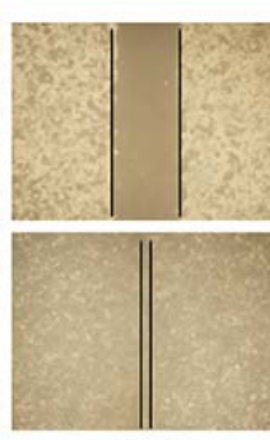

$50 \mu \mathrm{M}$

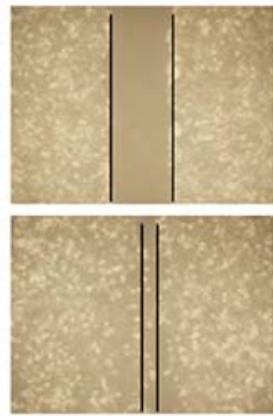

$50 \mu \mathrm{M}$

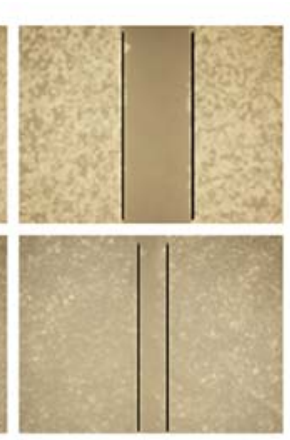

$75 \mu \mathrm{M}$

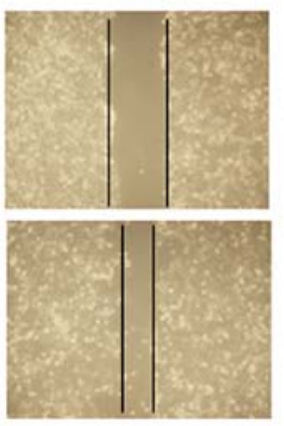

$75 \mu \mathrm{M}$
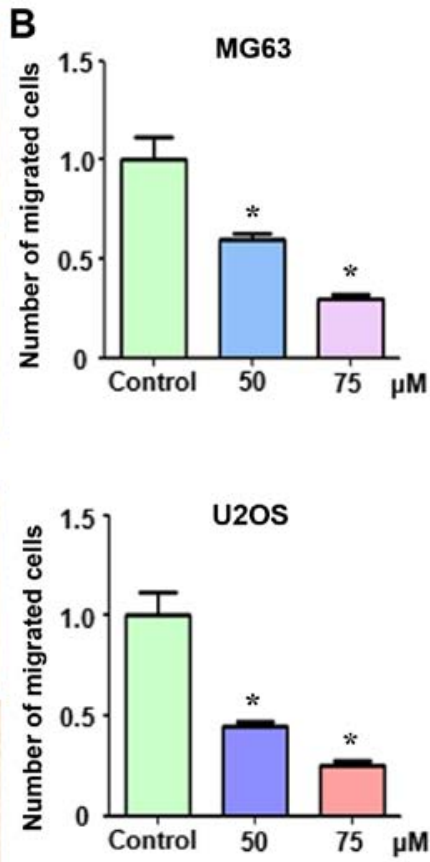

Figure 5. Apcin inhibits osteosarcoma cancer cell migration. (A) The migration ability of apcin-treated MG63 and U2OS cells was assessed using a wound healing assay. Briefly, MG63 and U2OS cells were seeded on a 6-well plate and incubated until the monolayer reached 90\% confluence. A rectangular wound was created using a 100- $\mu 1$ pipette tip. The open gap was inspected microscopically over time as cells migrated to fill the wound. Images were captured using an inverted microscope. (B) Quantitative results of A. $\mathrm{P}<0.05$ vs. the control group.

Apcin

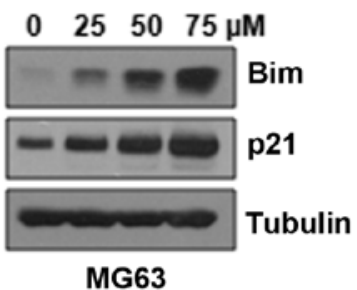

Apcin

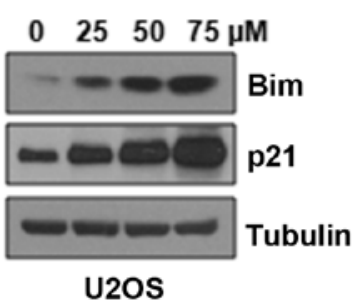

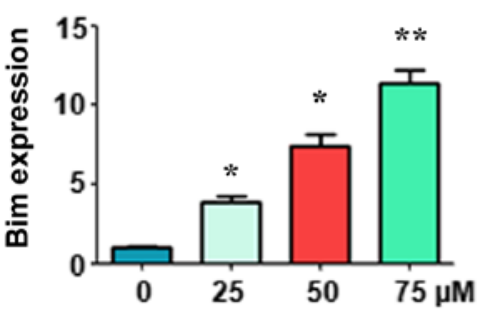
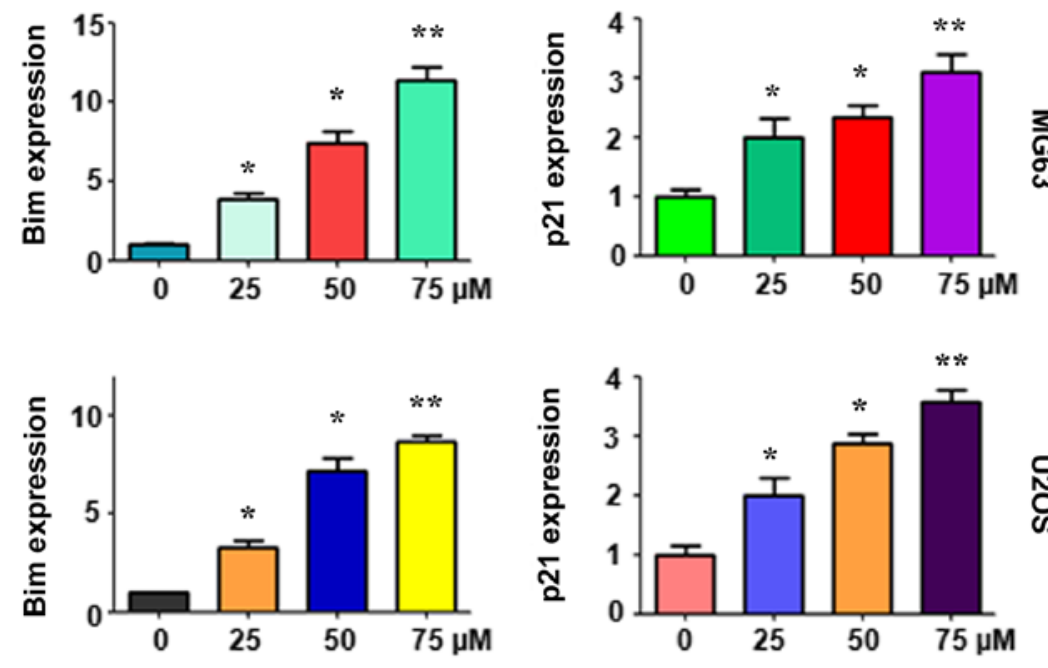

Figure 6. Bim and p21 are upregulated in MG63 and U2OS cells following apcin treatment. Left panel, the expression of Bim and its downstream target p21 in apcin-treated MG63 and U2OS cells was detected using western blotting. Right panel, quantitative results of immunoblots. ${ }^{*} \mathrm{P}<0.05$ and ${ }^{* *} \mathrm{P}<0.001$ vs. control.

Bim expression was induced by apcin treatment and whether it exhibited its antitumor activity in human OS cells. The results of western blotting demonstrated that the expression of Bim was increased in MG63 and U2OS cells following apcin treatment (Fig. 6). It was also observed that apcin promoted Bim expression in a dose-dependent manner. Furthermore, p21, a downstream target of Bim, was also significantly upregulated in a dose-dependent manner following apcin treatment (Fig. 6).
These findings revealed that the antitumor activity of apcin in OS cancer cells may be attributable, at least in part, to the increased expression of Bim and p21.

\section{Discussion}

Osteosarcoma (OS) is a malignant tumor of mesenchymal origin that primarily affects the long bones in children and 
young adults. Typically, it has a bimodal age distribution in the second decade of life and late adulthood $(3,29)$. At present, patients with low-grade OS lesions are typically treated with surgical resection alone. However, for high-grade lesions, neoadjuvant therapy followed by wide surgical resection of the tumor and a course of adjuvant chemotherapy is the typical treatment regimen. The use of adjuvant chemotherapy improves the 5-year survival rate of patients with OS from $<20$ to $\sim 70 \%(6,30)$. Neoadjuvant chemotherapy with high-dose methotrexate, doxorubicin, cisplatin and ifosfamide is commonly used to treat patients with OS. Other agents, including vincristine, bleomycin and cyclophosphamide, are sometimes used as well (31).

Previous studies have revealed that $\mathrm{Cdc} 20$ controls the substrate specificity of $\mathrm{APC} / \mathrm{C}$ to bind and ubiquitinate its target proteins for subsequent degradation. Cdc20 is often upregulated in tumors and is associated with clinicopathological parameters (13). For instance, high expression of Cdc20 was associated with higher tumor grades in bladder, cervical, colonic, endometrial, gastric, liver, ovarian, prostatic and renal carcinomas (32). There was a significant correlation between Cdc20 upregulation and advanced tumor stage in breast, colon, endometrium and prostate cancers (32). Furthermore, $\mathrm{Cdc} 20$ overexpression predicted a poor prognosis in a wide range of human malignant tumors (17-22,23), and thus may be used as a biomarker of tumor prognosis. As such, inhibiting Cdc20 expression or binding may serve as an effective cancer treatment. Inhibition of Cdc20 by its siRNA reduced the cell growth and invasion of OS cells (33). A number of Cdc20 inhibitors have been identified and extracted from natural compounds, including withaferin $\mathrm{A}$, which was demonstrated to modulate the spindle assembly checkpoint by degrading the Mad2-Cdc20 complex in colorectal cancer cells (34). Compound 331 was demonstrated to selectively induce glioma cell death by upregulating miR-494 and downregulating Cdc20 (35). Furthermore, rottlerin was demonstrated to inhibit cell growth and invasion by downregulating Cdc20 in glioma cells (36). It has also been reported that curcumin suppressed Cdc20 expression in pancreatic cancer cells (25). A number of natural compounds exhibit multiple non-specific targets and it would be beneficial to discover a specific Cdc20 inhibitor for cancer treatment. To this end, Zeng et al (37) demonstrated that pharmacologically disrupting the APC-Cdc20/Cdh1 interaction with tosyl-L-arginine methyl ester inhibited E3 ligase activity, inducing spindle checkpoint-dependent mitotic arrest in the absence of spindle damage. In addition, they identified another Cdc20-specific APC inhibitor, apcin, that was able to bind with Cdc20, block substrate recognition and inhibit the ubiquitination of Cdc20 substrates (26).

Apcin was initially reported as an inhibitor of cyclin proteolysis in mitotic Xenopus egg extract (38). Recently, Sackton et al (26) reported that apcin directly disturbed the binding of Cdc20 to its substrates and subsequently blocked mitotic exit in human cancer cells. Apcin inhibited the oncogenic function of $\mathrm{Cdc} 20$ by reducing cell viability and inducing apoptosis in a dose-dependent manner in prostate cancer cells (39). Furthermore, it was reported that Speckle-type POZ protein (SPOP)-deficient prostate cancer cells were resistant to apcin, suggesting that apcin could be clinically used to treat patients with SPOP-WT prostate cancer, but not
SPOP-deficient prostate cancer. It was reported that Warsaw breakage syndrome cell lines and several cancer cell lines with cohesion defects exhibited a significantly increased response to apcin (28). In the present study, it was demonstrated that apcin inhibited OS cell growth and induced significant apoptosis. The mobility of OS cells was also markedly inhibited by apcin treatment. Our findings revealed that apcin could have potential application in chemotherapy for OS patients.

A previous study reported that Cdc20 suppressed cell apoptosis largely via targeting Bim for polyubiquitination and degradation (40). Bim belongs to the Bcl-2-homology 3-only family (41). It has been reported that Bim interacts with $\mathrm{Bcl}-2, \mathrm{Bcl} 2 \mathrm{~L} 1$ and other $\mathrm{Bcl}-2$ proteins to act as an apoptotic activator under both physiological and pathological conditions to initiate the indigenous apoptotic pathway (41-44). Bim is widely expressed in a variety of tissues and regulates a number of normal and pathological systems (45). In the past few years, Bim has attracted more and more attention as a plausible target for cancer therapy (46). It has been reported that Bim plays a vital role in the anoikis of a number of cancer cells, including breast and lung cancer, fibrosarcoma, melanoma and OS (47-50). It was further demonstrated that aberrant levels of Bim affected chemotherapy response (51). For instance, Bim overexpression was revealed to enhance the efficacy of microtubule-targeting chemotherapy and may be a powerful predictor of tumor response to microtubule-targeting agents, including paclitaxel and vinorelbine (52). In contrast, Bim downregulation following siRNA transfection delayed paclitaxel-induced apoptosis, suggesting that low expression of Bim is responsible for paclitaxel-resistance in cancer cells (52). It has also been demonstrated that Bim deletion polymorphisms are associated with a poor clinical response to erlotinib and may be an independent prognostic factor for patients with non-small cell lung cancer with the EGFR mutation $(53,54)$. Collectively, these previous studies suggest that many chemotherapeutic agents use Bim to trigger apoptosis in a variety of cancer cells. Bim-targeting therapies may offer more effective and individual options for cancer treatment in the future.

A number of chemotherapeutic agents, including molecular-targeting agents imatinib, gefitinib and bortezomib, use Bim as an executioner. These agents could be classified as 'primitive' Bim-targeting agents (46). Recently, Gambichler et al (55) demonstrated that Bim protein expression was significantly inversely correlated with melanoma features that are associated with poor prognosis. They further reported that Bim was an independent predictor of advanced disease, confirming that this pro-apoptotic BH3-only protein may be a potent biomarker and promising therapeutic target. High Bim expression is a potential prognostic marker as well as a chemotherapeutic target for cervical cancer (56). The Bim deletion polymorphism was found to be associated with primary resistance to crizotinib in patients with ALK fusion-positive non-small cell lung cancer (57). As such, targeting and manipulating Bim expression or activity may affect the outcomes of human cancers. Pharmacological inhibition of APC/C with proTAME induced apoptosis in multiple myeloma cells and was partially mediated by Bim, as well as the phosphorylation of Bcl-2 and Bcl-xL (27). It was reported that Bim expression was upregulated by proTAME treatment, while the apoptotic consequences of $\mathrm{Cdc} 20$ depletion have 
been attributed to the accumulation of Bim. The results of the present study demonstrated that Bim was upregulated in OS cells following apcin treatment. Furthermore, apcin-induced Bim upregulation was dose-dependent. These results revealed that apcin exhibited its antitumor activity in OS cells. Bim overexpression may be due, at least in part, to disruption of the apcin-induced APC-Cdc20 interaction. As such, apcin may have potential as a treatment for human OS and Cdc20 may be a promising molecular target for chemotherapy.

\section{Acknowledgements}

Not applicable.

\section{Funding}

No funding was received.

\section{Availability of data and materials}

All data generated or analyzed during this study are included in this published article.

\section{Authors' contributions}

YG and GS were involved in the conceptualization of the study; YG, BZ, and YW were involved in data curation; YG and GS were involved in formal analysis; YG, BZ, YW, and GS were involved in the investigative aspects of the study; GS was involved in project administration and supervised the study; YG and GS wrote and edited the manuscript. All authors read and approved the manuscript and agree to be accountable for all aspects of the research in ensuring that the accuracy or integrity of any part of the work are appropriately investigated and resolved.

\section{Ethics approval and consent to participate}

Not applicable.

\section{Patient consent for publication}

Not applicable.

\section{Competing interests}

The authors declare that they have no competing interests.

\section{References}

1. Shaikh AB, Li F, Li M, He B, He X, Chen G, Guo B, Li D, Jiang F, Dang L, et al: Present advances and future perspectives of molecular targeted therapy for osteosarcoma. Int J Mol Sci 17: 506, 2016.

2. Zhou W, Hao M, Du X, Chen K, Wang G and Yang J: Advances in targeted therapy for osteosarcoma. Discov Med 17: 301-307, 2014.

3. Moore DD and Luu HH: Osteosarcoma. Cancer Treat Res 162: 65-92, 2014.

4. Jones KB: Osteosarcomagenesis: Modeling cancer initiation in the mouse. Sarcoma 2011: 694136, 2011.

5. Li X, Jiang H, Xiao L, Wang S and Zheng J: miR-200bc/429 inhibits osteosarcoma cell proliferation and invasion by targeting PMP22. Med Sci Monit 23: 1001-1008, 2017.
6. Allison DC, Carney SC, Ahlmann ER, Hendifar A, Chawla S, Fedenko A, Angeles C and Menendez LR: A meta-analysis of osteosarcoma outcomes in the modern medical era. Sarcoma 2012: 704872, 2012

7. Wu PK, Chen WM, Chen CF, Lee OK, Haung CK and Chen TH: Primary osteogenic sarcoma with pulmonary metastasis: Clinical results and prognostic factors in 91 patients. Jpn J Clin Oncol 39: 514-522, 2009.

8. Bielack SS, Kempf-Bielack B, Delling G, Exner GU, Flege S, Helmke K, Kotz R, Salzer-Kuntschik M, Werner M, Winkelmann W, et al: Prognostic factors in high-grade osteosarcoma of the extremities or trunk: An analysis of 1,702 patients treated on neoadjuvant cooperative osteosarcoma study group protocols. J Clin Oncol 20: 776-790, 2002.

9. Nandi D, Tahiliani P, Kumar A and Chandu D: The ubiquitin-proteasome system. J Biosci 31: 137-155, 2006.

10. Chang LF, Zhang Z, Yang J, McLaughlin SH and Barford D: Molecular architecture and mechanism of the anaphase-promoting complex. Nature 513: 388-393, 2014.

11. Passmore LA, McCormack EA, Au SW, Paul A, Willison KR, Harper JW and Barford D: Docl mediates the activity of the anaphase-promoting complex by contributing to substrate recognition. EMBO J 22: 786-796, 2003.

12. Sewart K and Hauf S: Different functionality of Cdc20 binding sites within the mitotic checkpoint complex. Curr Biol 27: 1213-1220, 2017.

13. Wang L, Zhang J, Wan L, Zhou X, Wang Z and Wei W: Targeting $\mathrm{Cdc} 20$ as a novel cancer therapeutic strategy. Pharmacol Ther 151: 141-151, 2015.

14. Paul D, Ghorai S, Dinesh US, Shetty P, Chattopadhyay S and Santra MK: Cdc20 directs proteasome-mediated degradation of the tumor suppressor SMAR1 in higher grades of cancer through the anaphase promoting complex. Cell Death Dis 8: e2882, 2017.

15. Marucci G, Morandi L, Magrini E, Farnedi A, Franceschi E, Miglio R, Calò D, Pession A, Foschini MP and Eusebi V: Gene expression profiling in glioblastoma and immunohistochemical evaluation of IGFBP-2 and CDC20. Virchows Arch 453: 599-609, 2008.

16. Mao Y, Li K, Lu L, Si-Tu J, Lu M and Gao X: Overexpression of Cdc20 in clinically localized prostate cancer: Relation to high Gleason score and biochemical recurrence after laparoscopic radical prostatectomy. Cancer Biomark 16: 351-358, 2016.

17. Choi JW, Kim Y, Lee JH and Kim YS: High expression of spindle assembly checkpoint proteins CDC20 and MAD2 is associated with poor prognosis in urothelial bladder cancer. Virchows Arch 463: 681-687, 2013.

18. Kim Y, Choi JW, Lee JH and Kim YS: MAD2 and CDC20 are upregulated in high-grade squamous intraepithelial lesions and squamous cell carcinomas of the uterine cervix. Int J Gynecol Pathol 33: 517-523, 2014.

19. Wu WJ, Hu KS, Wang DS, Zeng ZL, Zhang DS, Chen DL, Bai L and Xu RH: CDC20 overexpression predicts a poor prognosis for patients with colorectal cancer. J Transl Med 11: 142, 2013.

20. Chang DZ, Ma Y, Ji B, Liu Y, Hwu P, Abbruzzese JL, Logsdon C and Wang $\mathrm{H}$ : Increased CDC20 expression is associated with pancreatic ductal adenocarcinoma differentiation and progression. J Hematol Oncol 5: 15, 2012.

21. Moura IM, Delgado ML, Silva PM, Lopes CA, do Amaral JB, Monteiro LS and Bousbaa H: High CDC20 expression is associated with poor prognosis in oral squamous cell carcinoma. J Oral Pathol Med 43: 225-231, 2014.

22. Ding ZY, Wu HR, Zhang JM, Huang GR and Ji DD: Expression characteristics of CDC20 in gastric cancer and its correlation with poor prognosis. Int J Clin Exp Pathol 7: 722-727, 2014.

23. Shi R, Sun Q, Sun J, Wang X, Xia W, Dong G, Wang A, Jiang F and $\mathrm{Xu}$ L: Cell division cycle 20 overexpression predicts poor prognosis for patients with lung adenocarcinoma. Tumour Biol 39: 1010428317692233, 2017.

24. Li K, Mao Y, Lu L, Hu C, Wang D, Si-Tu J, Lu M, Peng S, Qiu J and GaoX: Silencing of CDC20 suppresses metastatic castration-resistant prostate cancer growth and enhances chemosensitivity to docetaxel. Int J Oncol 49: 1679-1685, 2016.

25. Zhang Y, Xue YB, Li H, Qiu D, Wang ZW and Tan SS: Inhibition of cell survival by curcumin is associated with downregulation of cell division cycle $20(\mathrm{Cdc} 20)$ in pancreatic cancer cells. Nutrients 9: E109, 2017.

26. Sackton KL, Dimova N, Zeng X, Tian W, Zhang M, Sackton TB, Meaders J, Pfaff KL, Sigoillot F, Yu H, et al: Synergistic blockade of mitotic exit by two chemical inhibitors of the APC/C. Nature 514: 646-649, 2014 
27. Lub S, Maes A, Maes K, De Veirman K, De Bruyne E, Menu E, Fostier K, Kassambara A, Moreaux J, Hose D, et al: Inhibiting the anaphase promoting complex/cyclosome induces a metaphase arrest and cell death in multiple myeloma cells. Oncotarget 7: 4062-4076, 2016.

28. de Lange J, Faramarz A, Oostra AB, de Menezes RX, van der Meulen IH, Rooimans MA, Rockx DA, Brakenhoff RH, van Beusechem VW, King RW, et al: Defective sister chromatid cohesion is synthetically lethal with impaired APC/C function. Nat Commun 6: 8399, 2015.

29. Wachtel M and Schäfer BW: Targets for cancer therapy in childhood sarcomas. Cancer Treat Rev 36: 318-327, 2010.

30. Bernthal NM, Federman N, Eilber FR, Nelson SD, Eckardt JJ, Eilber FC and Tap WD: Long-term results ( $>25$ years) of a randomized, prospective clinical trial evaluating chemotherapy in patients with high-grade, operable osteosarcoma. Cancer 118 5888-5893, 2012.

31. Maki RG: Ifosfamide in the neoadjuvant treatment of osteogenic sarcoma. J Clin Oncol 30: 2033-2035, 2012.

32. Gayyed MF, El-Maqsoud NM, Tawfiek ER, El Gelany SA and Rahman MF: A comprehensive analysis of CDC20 overexpression in common malignant tumors from multiple organs: Its correlation with tumor grade and stage. Tumour Biol 37: 749-762, 2016.

33. Shang G, Ma X and Lv G: Cell division cycle 20 promotes cell proliferation and invasion and inhibits apoptosis in osteosarcoma cells. Cell Cycle 17: 43-52, 2018.

34. Das T, Roy KS, Chakrabarti T, Mukhopadhyay S and Roychoudhury S: Withaferin A modulates the Spindle assembly checkpoint by degradation of Mad2-Cdc20 complex in colorectal cancer cell lines. Biochem Pharmacol 91: 31-39, 2014.

35. Zhang L, Niu T, Huang Y, Zhu H, Zhong W, Lin J and Zhang Y: Compound 331 selectively induces glioma cell death by upregulating miR-494 and downregulating CDC20. Sci Rep 5: 12003, 2015 .

36. Wang L, Hou Y, Yin X, Su J, Zhao Z, Ye X, Zhou X, Zhou L and Wang $Z$ : Rottlerin inhibits cell growth and invasion via down-regulation of Cdc20 in glioma cells. Oncotarget 7: 69770-69782, 2016.

37. Zeng X, Sigoillot F, Gaur S, Choi S, Pfaff KL, Oh DC, Hathaway N, Dimova N, Cuny GD and King RW: Pharmacologic inhibition of the anaphase-promoting complex induces a spindle checkpoint-dependent mitotic arrest in the absence of spindle damage. Cancer Cell 18: 382-395, 2010.

38. Verma R, Peters NR, D'Onofrio M, Tochtrop GP, Sakamoto KM, Varadan R, Zhang M, Coffino P, Fushman D, Deshaies RJ and King RW: Ubistatins inhibit proteasome-dependent degradation by binding the ubiquitin chain. Science 306: 117-120, 2004

39. Wu F, Dai X, Gan W, Wan L, Li M, Mitsiades N, Wei W, Ding Q and Zhang J: Prostate cancer-associated mutation in SPOP impairs its ability to target $\mathrm{Cdc} 20$ for poly-ubiquitination and degradation. Cancer Lett 385: 207-214, 2017.

40. Wan L, Tan M, Yang J, Inuzuka H, Dai X, Wu T, Liu J, Shaik S, Chen G, Deng J, et al: APC(Cdc20) suppresses apoptosis through targeting Bim for ubiquitination and destruction. Dev Cell 29: 377-391, 2014

41. Zheng JH, Viacava Follis A, Kriwacki RW and Moldoveanu T: Discoveries and controversies in BCL-2 protein-mediated apoptosis. FEBS J 283: 2690-2700, 2016.
42. O'Connor L, Strasser A, O'Reilly LA, Hausmann G, Adams JM, Cory S and Huang DC: Bim: A novel member of the Bcl-2 family that promotes apoptosis. EMBO J 17: 384-395, 1998

43. U M, Miyashita T, Shikama Y, Tadokoro K and Yamada M: Molecular cloning and characterization of six novel isoforms of human Bim, a member of the proapoptotic Bcl-2 family. FEBS Lett 509: 135-141, 2001

44. Correia C, Lee SH, Meng XW, Vincelette ND, Knorr KL, Ding H, Nowakowski GS, Dai H and Kaufmann SH: Emerging understanding of Bcl-2 biology: Implications for neoplastic progression and treatment. Biochim Biophys Acta 1853: 1658-1671, 2015.

45. Sionov RV, Vlahopoulos SA and Granot Z: Regulation of Bim in health and disease. Oncotarget 6: 23058-23134, 2015.

46. Akiyama T, Dass CR and Choong PF: Bim-targeted cancer therapy: A link between drug action and underlying molecular changes. Mol Cancer Ther 8: 3173-3180, 2009.

47. Tan TT, Degenhardt K, Nelson DA, Beaudoin B, Nieves-Neira W, Bouillet P, Villunger A, Adams JM and White E: Key roles of BIM-driven apoptosis in epithelial tumors and rational chemotherapy. Cancer Cell 7: 227-238, 2005.

48. Simpson CD, Anyiwe K and Schimmer AD: Anoikis resistance and tumor metastasis. Cancer Lett 272: 177-185, 2008.

49. Woods NT, Yamaguchi H, Lee FY, Bhalla KN and Wang HG: Anoikis, initiated by Mcl-1 degradation and Bim induction, is deregulated during oncogenesis. Cancer Res 67: 10744-10752, 2007.

50. Chen K, Tu Y, Zhang Y, Blair HC, Zhang L and Wu C: PINCH-1 regulates the ERK-Bim pathway and contributes to apoptosis resistance in cancer cells. J Biol Chem 283: 2508-2517, 2008.

51. Iurlaro R and Muñoz-Pinedo C: Cell death induced by endoplasmic reticulum stress. FEBS J 283: 2640-2652, 2016.

52. Savry A, Carre M, Berges R, Rovini A, Pobel I, Chacon C, Braguer D and Bourgarel-Rey V: Bcl-2-enhanced efficacy of microtubule-targeting chemotherapy through Bim overexpression: Implications for cancer treatment. Neoplasia 15: 49-60, 2013.

53. Cardona AF, Rojas L, Wills B, Arrieta O, Carranza H, Vargas C, Otero J, Corrales-Rodriguez L, Martín C, Reguart N, et al: BIM deletion polymorphisms in Hispanic patients with non-small cell lung cancer carriers of EGFR mutations. Oncotarget 7: 68933-68942, 2016.

54. Costa C, Molina MA, Drozdowskyj A, Giménez-Capitán A, Bertran-Alamillo J, Karachaliou N, Gervais R, Massuti B, Wei J, Moran T, et al: The impact of EGFR T790M mutations and BIM mRNA expression on outcome in patients with EGFR-mutant NSCLC treated with erlotinib or chemotherapy in the randomized phase III EURTAC trial. Clin Cancer Res 20: 2001-2010, 2014.

55. Gambichler T, Rooms I, Scholl L, Stockfleth E, Stucker M and Sand M: BH3-only protein Bim predicts advanced stage of cutaneous melanoma. J Eur Acad Dermatol Venereol 30: 1926-1929, 2016.

56. Kim BW, Cho H, Ylaya K, Kitano H, Chung JY, Hewitt SM and $\mathrm{Kim} \mathrm{JH}$ : Bcl-2-like protein 11 (BIM) expression is associated with favorable prognosis for patients with cervical cancer. Anticancer Res 37: 4873-4879, 2017.

57. Zhang L, Jiang T, Li X, Wang Y, Zhao C, Zhao S, Xi L, Zhang S, Liu X, Jia Y, et al: Clinical features of Bim deletion polymorphism and its relation with crizotinib primary resistance in Chinese patients with ALK/ROS1 fusion-positive non-small cell lung cancer. Cancer 123: 2927-2935, 2017. 